\title{
NeuroRegulation
}

\section{EEG Neurofeedback in the Treatment of Chronic Pain: A Case Series}

\author{
Edward H. Jacobs ${ }^{1 *}$ and Mark P. Jensen ${ }^{2}$ \\ ${ }^{1}$ Londonderry, New Hampshire, USA \\ ${ }^{2}$ University of Washington, Washington, USA
}

\begin{abstract}
Neurofeedback has been used to treat a variety of problems and symptoms related to central nervous system dysregulation, including chronic pain conditions. However, there is limited published work describing the application and efficacy of neurofeedback for chronic pain. This case series describes the outcomes of neurofeedback treatment of four patients with diverse diagnoses and pain symptoms. Although there was variability in patient response, all patients reported improvements in pain and other symptoms with treatment. The findings indicate that more research to (1) clarify the benefits of neurofeedback for different conditions and (2) identify the most effective protocols for individual patients is warranted.
\end{abstract}

Keywords: Neurofeedback; chronic pain; migraine; gastrointestinal pain; testicular pain

Citation: Jacobs, E. H., \& Jensen, M. P. (2015). EEG Neurofeedback in the Treatment of Chronic Pain: A Case Series. NeuroRegulation, 2(2), 86-102. http://dx.doi.org/10.15540/nr.2.2.86

*Address correspondence to: Edward $\mathrm{H}$. Jacobs, PhD, 12 Parmenter

Road, Londonderry, NH 03053, USA. Email: ehjpsych@aol.com

Edited by:

Randall Lyle, PhD, Mount Mercy University, lowa, USA

Copyright: @ 2015. Jacobs and Jensen. This is an Open Access article distributed under the terms of the Creative Commons Attribution License (CC-BY).

Reviewed by:

Randall Lyle, PhD, Mount Mercy University, lowa, USA

Sarah Prinsloo, PhD, The University of Texas, MD Anderson Cancer Center, Texas, USA

\section{Introduction}

Neurofeedback procedures, by which individuals are trained, through operant conditioning, to increase or inhibit the magnitude ("power") of oscillations in specific bandwidths as a way to self-regulate brain activity, have been used to improve arousal, alertness, emotional control, and symptom expression (Othmer, Othmer, \& Kaiser, 1999); symptoms of attention deficit disorder (Arns, de Ridder, Strehl, Breteler, \& Coenen, 2009; Linden, Habib, \& Radojevic, 1996; Lubar, 1991; Monastra, Monastra, \& George, 2002); learning and cognitive functioning (Albert, Andrasik, Moore, \& Dunn, 1998; Cunningham \& Murphy, 1981; Egner \& Gruzelier, 2003; Fenger, 1995; Gruzelier, Egner, \& Vernon, 2006; Murphy, Darwin, \& Murphy, 1977; Nall, 1973; Rasey, Lubar, Mclntyre, Zoffuto, \& Abbott, 1996; Thompson \& Thompson, 1998; Vernon et al., 2003; Whisenant \& Murphy, 1977); and artistic performance (Gruzelier, 2009).

One class of symptoms to which neurofeedback has been applied is chronic pain (Jensen, Sherlin, Hakimian, \& Fregnia, 2009). Chronic pain is defined with respect to acute pain by the National Institute of Neurological Disorders and Stroke (NINDS) of the National Institutes of Health (2015) as follows:

While acute pain is a normal sensation triggered in the nervous system to alert you to possible injury and the need to take care of yourself, chronic pain is different. Chronic pain persists. Pain signals keep firing in the nervous system for weeks, months, even years. There may have been an initial mishap-sprained back, serious infection, or there may be an ongoing cause of painarthritis, cancer, ear infection, but some people suffer chronic pain in the absence of any past injury or evidence of body damage (para. 1).

Although early theories of chronic pain focused more on the periphery as the site of presumed physical damage that is likely "causing" pain, current models of chronic pain acknowledge the critical role that the brain plays in creating the pain experience. As a result, there has been an increased interest in 
treatments that might impact pain via their direct effects on brain activity, including neurofeedback.

Consistent with the possibility that neurofeedback could benefit individuals with chronic pain, a growing body of evidence supports pain perception as being mediated by structures and neural networks in the brain and as being influenced by multiple, interactive neural processes that modulate pain information at many levels, including the cortex. Multiple cortical sites have been identified as involved, including the somatosensory cortex, the insular cortex, the anterior cingulate, the prefrontal cortex, and thalamic nuclei (Apkarian, Bushnell, Treede, \& Zubeita, 2005; Babiloni et al., 2003; Chen, 2001; Craig, 2003a, 2003b; DeCharmes et al., 2005; DeLeo, 2006; Jensen, 2010; Katz \& Rothenberg, 2005; Melzack, Coderre, Katz, \& Vaccarino, 2001; Miltner \& Weiss, 1998; Peyron, Laurent, \& Garcia-Larrea, 2000; Rainville, Duncan, Price, Carrier, \& Bushnell, 1997; Tinazzi, Fiaschi, Rosso, Faccioli, Grosslercher, \& Aglioti, 2000). Furthermore, there is evidence of neural adaptation to pain stimuli, indicating neural plasticity (Flor, 2003; Katz \& Melzack, 1990). Consistent with this idea, repeated exposure to painful stimuli has been shown to increase one's sensitivity to stimulation and, therefore, to the tendency to interpret stimulation that may not be damaging as "pain," contributing to chronicity (Bromm \& Lorenz, 1998). The pain circuitry in the brain also overlaps the circuitry involved in depression, providing further evidence of central nervous system involvement in pain modulation (Lindsay \& Wyckoff, 1981).

A number of studies have identified specific frequencies of brain wave activity that are associated with pain. For example, the presence of more activity in the alpha frequency range $(8-13 \mathrm{~Hz})$ is known to be associated with, and to reflect a general inhibition of, cognitive activity and central nervous system (CNS) processing (Pfurtscheller, 2003); hence, lower alpha activity has been associated with increased pain perception and higher alpha activity with decreased pain perception (Babiloni et al., 2008; Nishigami, Nakano, Osumi, Tsujishita, Mibu, \& Ushida, 2014). Because neurons and neuronal ensembles that fire in the alpha frequency tend to inhibit activity in "downstream" neurons that are influenced by these ensembles, lower alpha power is associated with more information processing, including information that is transferred through thalamo-cortical and corticocortical channels. Suppression of the alpha rhythm could therefore allow for more processing of nociceptive input from the periphery. Consistent with this idea, suppression of alpha power (also known as alpha event-related desynchronization) has been found to occur in the primary somatosensory cortex in anticipation of aversive or painful electrical stimulus (Burroughs, 2011). Lower alpha activity has also been implicated in the perception of pain accompanying sensorimotor incongruent information (Nishigami et al., 2014) and in the anticipation of painful motor stimuli (Babiloni et al., 2008). Thus, it is reasonable to hypothesize that the stronger the magnitude of event-related desynchronization of alpha in anticipation of pain, the greater the subjectively rated experience of pain. In addition, research has shown that individuals with chronic pain exhibit increased beta and decreased alpha CNS activity, with additional increased theta activity in individuals with spinal cord injury and chronic pain (Jensen et al., 2009).

Consistent with this research linking brain oscillation patterns to the severity of pain, a number of studies have demonstrated improvements in pain sensation/perception in various pain conditions following neurofeedback training (Jensen et al., 2009; Prinsloo, Gabel, Lyle, \& Cohen, 2012). However, there are important gaps in our knowledge regarding the potential of neurofeedback for treating pain. For example, there is not yet a sufficient empirical or theoretical basis for deriving protocols from a common understanding of the specific brain oscillations to reward and inhibit. We also do not yet know if the protocols that can be derived from the limited understanding we do have would be equally effective across different pain conditions. Moreover, questions remain regarding the adaptability of such protocols to the realities of an outpatient clinical practice, in which there is less an emphasis on standardization than on the ability to modify the treatment approach in response to clinical considerations, such as client response. Such tailoring of training protocols has been a recent focus for researchers (Arns, Conners, \& Kraemer, 2013; Arns, Heinrich, \& Strehl, 2014; Escolano, Navarro-Gil, Garcia-Campayo, Congedo, \& Minguez, 2014; Lansbergen, van DongenBoomsma, Buitelaar, \& Slaats-Willemse, 2011; Logemann, Lansbergen, Van Os, Böcker, \& Kenemans, 2010).

Given these considerations, the aim of this paper is to provide additional information regarding how neurofeedback training might be used for treating individuals with chronic pain in the context of an active clinical practice. This information could be useful to clinicians by providing them with specific procedures and treatment protocols to consider in 
patients they treat with chronic pain. The information could also be useful to researchers by providing them with specific treatment protocols to evaluate in future clinical trials.

\section{Methods}

\section{Patient selection}

The subjects of this case series were four consecutive patients in the outpatient practice of the first author who met the following criteria: (1) presenting with a primary complaint of chronic pain, (2) not presenting with severe psychological problems other than anxiety and/or depression. Thus, the participants were not selected based on their response to treatment. The patients were screened using a comprehensive intake interview without the use of standardized psychological measures.
Intake interviews

Each patient first underwent an initial 45-min intake interview in which the patient's presenting problems, current functioning, and psychosocial and medical histories were taken. This was followed by a second intake session, which consisted of a 45-min introduction to the equipment and procedures of the neurofeedback training. Baseline levels of the outcome variables were assessed during the second session, and monitored throughout treatment, using a Neurofeedback Progress Chart (NPC; Matthew Fleischman, PhD, The Center for Attention and Learning, Eugene, OR) as shown in Figure 1. With the NPC, patients are told to describe each problem or symptom they would like to see improve with treatment, and then asked to rate the severity of each one by circling a number on a 0-4 Likert scale $(0=$ Not at all a problem to $4=$ Very much a problem). They were also told that they could place a circle between the numbers, which essentially turned the measure into a 9-point scale.

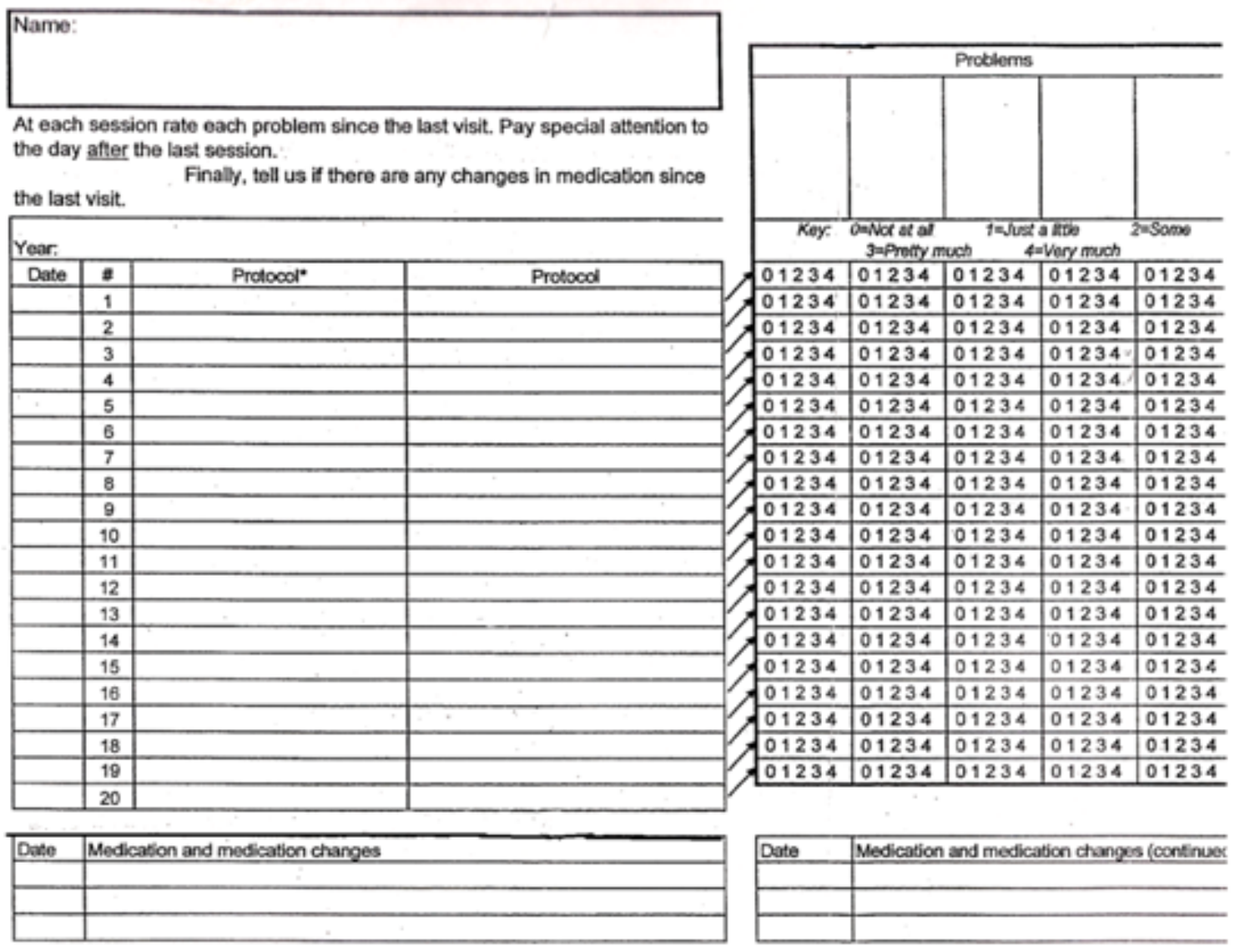

Figure 1. Neurofeedback Progress Chart. 


\section{Equipment}

Training was conducted using software developed by EEG Spectrum International (later called EEG Education and Research; Granada Hills, CA) version 4.30. The software was run on a single computerdual monitor system, with one Dell Inspiron 620, i52310, $2.90 \mathrm{GHz}, 64-$ bit desktop computer and two Optiquest monitors. The signal was processed with a Thought Technology ProComp+ (Quebec, Canada) amplifier, and 16" silver electrodes were applied to the scalp and ears with a preparation of NuPrep gel and Ten20-Conductive paste with an impedance of $\leq 20 \mathrm{kohm}$. The patient received visual and auditory feedback through the display of a "game" interface. The visual and auditory feedback was responsive to the patient simultaneously increasing the microvoltage reading of a specified frequency band of brain oscillation activity ("reward band") and decreasing the microvoltage reading of two other specified frequencies of brain wave activity ("inhibit bands"), above or below their respective thresholds, which were programmed into the computer by the clinician before each treatment session. The thresholds were also adjusted at times during each treatment session to increase the level of difficulty for the patient when the patient's success resulted in the training being much easier than previously.

\section{Training procedure}

At the beginning of each neurofeedback training session, beginning with the second session, the patient was presented with the NPC and instructed to: "...complete the ratings for each symptom or problem based on how much of a problem it has been for you since the last session, with particular emphasis on the day or two immediately after the last session."

Electrodes were placed on the patient's scalp and earlobes after prepping the skin. Electrode placement was made according to the standard 1010 electrode placement system in which electrode sites are identified with a letter and a number (see Figure 2).

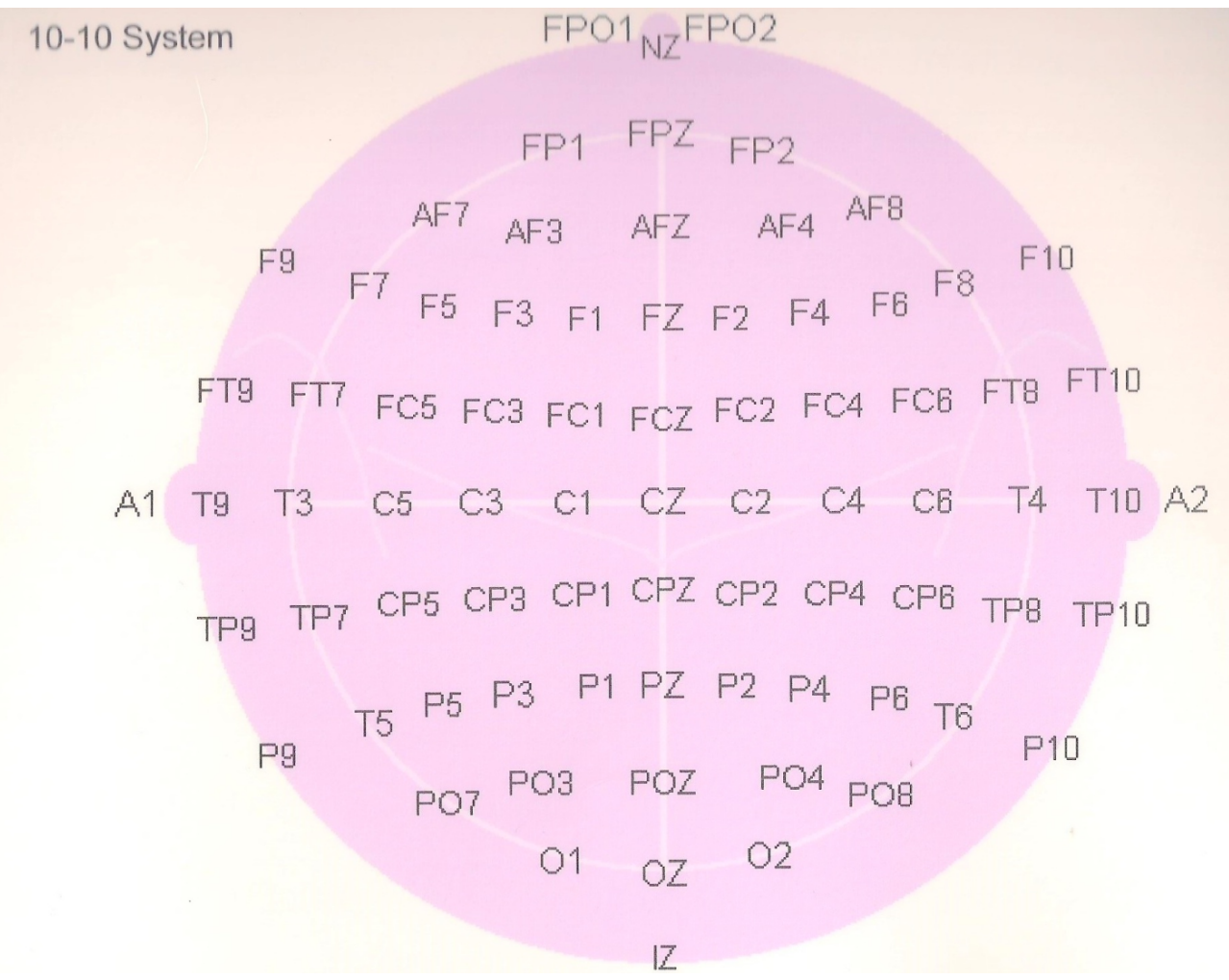

Figure 2. 10-10 Electrode Placement System. 
Three electrodes were placed for each protocol: one for the active training site, one for the reference site, and one for ground. The first site was the active site and always involved placement somewhere on the scalp over a cortical region that was the target of training. The second (reference) electrode was placed either at another scalp location (in bipolar training) or on an earlobe (in unipolar training). The third electrode was placed on what was considered an electroencephalography (EEG) neutral site, such as an earlobe, as a ground for the amplifier. Placements are identified by the active site followed by the reference (e.g., C4-A2). All treatment protocols were determined by the clinician and at the time of patient presentation or during the course of treatment. Given the authors' interest in determining the efficacy of electrode sites and bandwidths cited in the literature (e.g., Jensen et al., 2009), treatment protocols were based on this literature rather than on quantitative EEG (qEEG) findings.

During treatment, the patients were seated upright in a chair facing the computer monitor, which displayed the game or the interface. The therapist sat on the patient's left, facing the screen, which displayed the patient's EEG activity. The patient was instructed to "Sit comfortably, relax and focus." If the patient asked what he or she was supposed to "do" to play the game, he or she was reassured using words similar to the following: "Just relax all of your muscles, relax your jaw and your neck, and get into a state of relaxed focus. Your job is to [language inserted appropriate to what would happen if the patient earned points in the game, such as '...keep the circle moving through the maze eating dots']. Your brain will learn from the feedback."

For all patients, the thresholds were set to begin at $20-60-15$, where 20 referred to the percentage of time that the microvoltage of the lower frequency inhibit band exceeded the threshold, making the patient ineligible for a reward (visual or auditory), 60 referred to the percentage of time that the microvoltage of the reward band exceeded the threshold, making the patient eligible for a reward, and 15 referred to the percentage of time that the microvoltage of the lower frequency inhibit band exceeded the threshold, making the patient ineligible for a reward. In order for the patient to receive a visual and auditory reward, all three threshold criteria had to be met simultaneously. The thresholds were adjusted throughout the training to control for the level of difficulty of reaching criteria for reward in order to balance the need for an appropriate level of challenge (i.e., not make meeting the training criterion too easy, reducing the opportunity for learning) with the need to make meeting the training criterion possible (i.e., to limit anxiety and frustration). This was a clinical decision made individually for each patient.

When a patient sits down to begin a neurofeedback training session, there is often a shift in the amplitude of the EEG activity. In order to start the training session at the appropriate level of difficulty (with the first inhibit band starting out at $20 \%$ above threshold, the reward band at $60 \%$ above threshold, and the second inhibit band at $15 \%$ above threshold), the therapist autothresholded the computer about 30 seconds after the patient first began training. The therapist remained in the room for the entire session, occasionally (but conservatively) adjusting the thresholds. Each training session lasted for $30 \mathrm{~min}$, with at least 18 of those minutes spent in actual neurofeedback training.

\section{Results}

\section{Case 1}

Presenting problem

A 19-year-old female high school senior presented with chronic abdominal pain, which she described as "sharp," "pulsing," and "aching." On a 1-10 pain scale, where 1 represented No pain, and 10 represented The worst pain you have ever experienced, she rated her typical daily pain at an 8 . In addition to severe pain, she complained of depressed mood, excessive sleep, and diminished energy and motivation. The patient was a high school senior and, for the week prior to her initial appointment, she had been out of school the entire week. She did not believe that she would feel well enough to attend the end of senior year social activities that had been planned.

\section{History}

When the patient was 13-years-old, she became ill with gastrointestinal symptoms-gastrointestinal pain and diarrhea-while at an overnight summer camp. Her symptoms persisted into the fall of that year, and she missed a large portion of 8th grade. At age 14, she experienced painful menstruation secondary to endometriosis and was prescribed Lupron and Prempro. At age 15, after swimming in a lake in the summer, she contracted a waterborne parasite (microsporidium) and developed stomach pain and diarrhea. These symptoms never resolved. Starting in the 8th grade, and continuing to the time she began treatment, the patient often missed weeks of school at a time. She also described a long history of anxiety and depression associated 
with the pain, and reported that, when these psychological symptoms became worse, so did her pain.

The patient had been treated at the Mayo Clinic and at Massachusetts General Hospital. She had been prescribed paroxetine, sertraline, escitalopram oxalate, duloxetine hydrochloride, and pregabalin, the latter two of which she was taking at the time of treatment. She also took melatonin to help her sleep. She had seen a therapist for cognitivebehavior therapy to help her cope with the pain. A neuropsychological evaluation in 2009 found her to be of average to above average intelligence and also diagnosed her with Attentiondeficit/Hyperactivity Disorder (ADHD). Prior to treatment, the patient completed the NPC and identified the following two areas of concern: Pain (rated as a 4 at pretreatment) and depression (rated as a 3).

\section{Course of treatment}

The patient was then seen for 41 neurofeedback sessions over an 8-month period. She was seen twice weekly for approximately 16 weeks, once every 2 weeks for approximately 12 weeks after that, and then once a month for approximately 9 weeks.

For sessions 1-3, a single channel, bipolar protocol was used with a ground to the right earlobe (A2). The bipolar protocol was T3-T4 with the active electrode at T3 and the reference electrode at T4. This protocol was chosen because of clinical reports of its effectiveness in emotional stabilization (Othmer, 2005). Various $3 \mathrm{~Hz}$-wide reward bands between 9-12 Hz (alpha) and 12-15 Hz (low beta) were used, out of interest in determining if a particular reward band would yield a fast, positive response. The inhibits used were 2-7 Hz (theta) and $22-32 \mathrm{~Hz}$ (fast beta). At the end of the three sessions, the symptom ratings remained the same. In the clinician's experience, patients often experience some subjective sense of improved wellbeing during the session at some frequency setting with this protocol. Because the patient did not experience any subjective improvements and was in a good deal of distress, the decision was made to switch to a different protocol in order to determine if the patient could experience some immediate relief.

Sessions 4-5 were conducted using a single channel, unipolar protocol, with the electrode either at $\mathrm{CZ}$ or $\mathrm{FZ}$, with the reference electrode on the left earlobe (A1) and the ground on the right earlobe (A2). The $C Z$ and $F Z$ sites were chosen for two reasons. First, they are over the anterior cingulate cortex, known to be important in the affective experience of pain (Rainville et al., 1997). Second, research has indicated that training at these sites is effective for reducing inattention symptoms in individuals who have a diagnosis of ADHD (Arns et al., 2009). The reward frequencies varied within the beta range in $3 \mathrm{~Hz}$-wide bands, ranging from 12-15 $\mathrm{Hz}$ to $20-23 \mathrm{~Hz}$, in order to find a frequency which felt most comfortable to her, particularly one in which she felt most focused, with the inhibits at 4-7 Hz and 22-32 Hz. No changes in the problem symptoms were reported following this protocol.

Sessions 6-10 were conducted with the unipolar placement at FZ-A1, with a reward of $8-13 \mathrm{~Hz}$, and a second, simultaneous, reward in the beta band, ranging between 12-15 Hz and 21-24 Hz. The alpha reward band was expanded by $1 \mathrm{~Hz}$ in both directions in order to capture more of the alpha spectrum. The inhibits remained at 4-7 Hz and 22$32 \mathrm{~Hz}$ during these sessions. Significant improvement was noted in both symptoms following these sessions, with pain reducing to a 2 and depression to a 1 .

Given these improvements, training at FZ-A1 continued for sessions 11-18, with rewards at 8-13 $\mathrm{Hz}$ and $12-15 \mathrm{~Hz}$, and inhibits at 4-7 $\mathrm{Hz}$ and 22-32 $\mathrm{Hz}$. The 12-15 Hz reward was added because of aforementioned research indicating that enhancements of alpha and slow beta frequencies could be effective in chronic pain treatment. Following these sessions, the previous reduction was maintained at a 2 and depression dropped further to a 0 .

For sessions 19-20, an attempt was made to train posteriorly, because pain had not improved as much as depression had, and because posterior training was thought to be consistent with the somatosensory nature of the pain. Four studies have reported positive outcomes with training that included occipital electrode placements (Andreychuk \& Skriver, 1975; Cohen, McArthur, \& Rickles, 1980; Gannon \& Sternbach, 1971; Melzack \& Perry, 1975) and two reported positive outcomes with training that included parietal placements (Cohen et al., 1980; Jensen, Grierson, Tracy-Smith, Bacigalupi, \& Othmer, 2007). A unipolar O2-A2 protocol rewarding $8-13 \mathrm{~Hz}$ and inhibiting $4-7 \mathrm{~Hz}$ and 22-32 $\mathrm{Hz}$ was used first, followed by a unipolar P4-A2 protocol with the same rewards and inhibits. Following these two training sessions, the patient reported a worsening of her symptoms, with pain increasing to a 3 and depression to a 4 . 
In an effort to return to the previous levels of improvement, the decision was made to return to training at the FZ-A1 site for session 21, with the rewards at $8-13 \mathrm{~Hz}$ and $12-15 \mathrm{~Hz}$, in order to separately reinforce alpha and slow beta activity, and the inhibits at $2-7 \mathrm{~Hz}$ and $22-32 \mathrm{~Hz}$. The decision to broaden the band of the slow wave inhibit was made because a significant amount of activity was observed by visual analysis in that part of the spectrum. The pain rating remained at 3 . The decision was made to switch to unipolar training at C4-A2 in order to see if using a protocol that has been linked to calming rather than activating the nervous system might be effective. Sessions 22-29 were conducted at that site, with rewards being 8-13 $\mathrm{Hz}$ and $12-15 \mathrm{~Hz}$, and inhibits being at 2-7 Hz and 22-32 Hz. The symptom ratings improved back to their previous lower levels, with pain rated as a 2 and depression a 0.

With the previous level of symptom improvement reestablished, sessions 30-33 employed a twochannel set up at the following sites: F1-A1 and F2A2, as suggested in Jensen et al. (2009), based on research indicating a role for the dorsal anterior cingulate in the affective experience of pain (Rainville et al., 1997). This was conducted with a reward band of $8-13 \mathrm{~Hz}$ and inhibits at $4-7 \mathrm{~Hz}$ and $15-32 \mathrm{~Hz}$, setting a broader fast frequency inhibit in order to address possible overarousal problems that might be contributing to the patient's pain symptoms. Following these sessions, the pain rating reduced further (to a 1) and the low level of depression (at a $0)$ maintained.

Because of the improvement in pain intensity, we continued with the same training sites for the final six sessions (sessions 34-41), with one change in the protocol: the slow wave inhibit band was further widened to $0-7 \mathrm{~Hz}$ because of the high level of activity observed through visual inspection of the single $\mathrm{Hz}$ display on the therapist monitor. Following these sessions, the symptom ratings were 0 for both problems.

Summary of treatment and behaviors outside of the treatment sessions

After the first four sessions, there was no change in the symptom ratings, and the patient reported experiencing a slight increase in pain and depression. Starting with session 5, the patient reported substantial decreases in pain and depression. She also reported having more energy, having improved sleep, and feeling more relaxed. After session 17, the patient took her GED (she had missed most of the end of her senior year), and was planning to attend senior prom. After session 22, the patient started looking for a job. She also reported that she started having periods of reduced pain outside of the sessions rather than constant severe pain. Following session 24, the patient started working 2 days per week. Through sessions 22-30, the patient experienced "flare ups" of her pain, but she reported that these were not as severe as her pain intensity before treatment, and felt manageable to the patient. After session 39, the patient started college and enrolled in three classes. At session 41, the patient reported that she was getting As in all of her classes and had registered for classes for the next semester.

\section{Case 2}

Presenting problems

A 14- year-old male ninth grade boy presented with chronic left testicular pain, which he experienced as an aching, and sometimes "surging" pain in his left testicle. He was taking anti-inflammatories, pain medication, and anti-depressant medication. $\mathrm{He}$ rated his daily pain as between 4 and 6 on a 1-10 numerical rating scale, even when he was taking pain medication. At intake, the patient was taking fluoxetine $40 \mathrm{mg}$. and tramadol $50 \mathrm{mg}$. The pain was worse with physical activity, including walking between classes in school. The patient attended a high school that consisted of several buildings on a campus, so walking between classes sometimes involved walking between buildings. Because of the pain, the patient had been attending school for halfdays for the past 6 months and had stopped sports activities (basketball and snowboarding) that he used to enjoy. The patient completed the NPC and identified a single area of concern-pain (rated as a 4 at pretreatment). EEG recordings over several cortical sites indicated higher than average beta activity over the posterior sites $\mathrm{O} 1$ and $\mathrm{O} 2$, and F4 beta power clearly greater than F3 beta.

\section{History}

About one year prior to intake, the patient experienced sharp pain in his left testicle while in gym class. He described the pain as the worst pain he had ever experienced, which made him cry. A urological examination indicated blood in his urine and he was diagnosed with epididymitis at Children's Hospital Boston.

\section{Course of treatment}

The patient was seen for 22 neurofeedback sessions approximately twice a week over a 3month period. For sessions $1-11$, the following twochannel protocol was used: O1-A1 and O2-A2, 8-11 $\mathrm{Hz}$ and $10-13 \mathrm{~Hz}$ rewards, and $4-7 \mathrm{~Hz}$ and $15-22$ 
$\mathrm{Hz}$ inhibits. Occipital placements were attempted both because these placements had been used successfully in mixed chronic pain conditions (Melzack \& Perry, 1975) and because of the high beta activity at those regions. Following session 3 , he rated his pain problem as 2 . Following session 7 and through session 11, he rated his pain problem as 1 .

The patient was then seen for sessions 12-22 with the training site being F4-A2, because of the high pretreatment beta activity at that site, with only a 15$22 \mathrm{~Hz}$ inhibit and no reward frequency band. By session 17 through the end of treatment, his rating of the pain problem dropped to 0 .

\section{Summary of treatment and behaviors outside of} the treatment sessions

After session 2, the patient reported that he had exerted himself more than usual outside of the office; specifically, he attended graduation parties and started playing basketball again. $\mathrm{He}$ experienced less pain when he played basketball and he did not ice himself after these activities, as he had been doing after any physical activity, including walking, before treatment. After session 11 , the patient played more rigorous basketball and reported that this did not increase his pain, as it had prior to treatment. At session 13, the patient reported that he had stopped experiencing random bursts of pain unrelated to activity. After session 14, the patient reported that he had played basketball and swam with little pain. He decided to discontinue fluoxetine. After session 15, the patient reported that he felt "grumpy" off of fluoxetine, so he resumed taking it. After session 16, the patient reported that he had experienced no pain except for experiencing a sharp pain with no precipitating event, lasting 1520 minutes once a week, for which he took pain medication. At session 18, the patient reported that his pain had disappeared and that he had gone jet skiing and had played basketball with no pain. At session 21, the patient reported that he had been able to walk around his school with no pain. He had experienced one incident of pain after school, for which he had taken pain medication.

\section{Case 3}

Presenting problem

A 56-year-old married woman presented with migraine headaches, which had been occurring five times a week. The headaches often lasted all day. Her migraines could get triggered by lifting her head off her pillow at night, turning in her bed, or getting out of bed. She was also experiencing anxiety and depression, which were triggered by family problems. In addition to mood disturbance, her symptoms included teeth clenching, crying, overeating, delayed sleep onset, and poor sleep maintenance. She also complained of memory problems. She was taking a combination analgesic containing acetaminophen, butalbital, and caffeine. The patient identified the following six areas of concern:

- migraine (rated as a 4),

- physical anxiety symptoms (by which she meant perceived muscle tension associated with anxiety, rated as a 4),

- depression (rated as a 4),

- $\quad$ sleep (rated as a 4),

- overeating (rated as a 4), and

- organizational skills (rated as a 3).

History

The patient reported that she had been experiencing migraines at the same frequency since she was 18 years old. She also experienced a great deal of stress raising her four children, all of whom she was homeschooling.

\section{Course of treatment}

The patient was seen in neurofeedback treatment twice weekly for 32 sessions. For sessions $1-5$, a bipolar placement at T3-T4 was used, because her migraines and the attendant mood dysregulation were seen as signs of an unstable arousal pattern (Othmer, 2005). Because the patient often experienced pain during the sessions, the inhibits and rewards were frequently changed during the sessions in order to determine if the patient could experience an immediate, positive response to training at specific frequencies. Slow inhibits varied (2-7 Hz, 6-9 Hz, 7-10 Hz, 8-11 Hz), and the reward band varied as well $(7-10 \mathrm{~Hz}$ to $12-15 \mathrm{~Hz}$ in $3-\mathrm{Hz}$ wide bands). There was a constant inhibit of 22-32 $\mathrm{Hz}$. The patient had difficulty controlling fast beta (22-32 Hz) activity and keeping it under threshold. She also reported a great deal of stress and guilt about family matters, and some time was spent engaging in problem solving with the patient on these matters at the beginning and end of each session, as she was being prepared for the neurofeedback training.

The patient's headaches varied in intensity during the sessions. To help address this, the patient was taught diaphragmatic breathing and was trained to increase Heart Rate Variability (HRV; McCraty, Atkinson, \& Tomasino, 2001) with the assistance of a computer software program installed on a laptop computer (emWave PC Stress Relief System, 
www.heartmath.com) on the fourth session, HRV was monitored simultaneously on the EEG feedback and the HRV equipment during this and most subsequent sessions. Following the fourth session, the migraine problem was rated as 2 , physical anxiety as 2 , depression was rated as 1 , sleep as 2 , overeating as 0 and organizational skill problem as 2.

Sessions 6-7 combined the following protocols: T3T4 with $2-7 \mathrm{~Hz}$ and $22-32 \mathrm{~Hz}$ inhibits and a $7-10 \mathrm{~Hz}$ reward, and FZ-A1 with a 22-32 Hz inhibit and a reward of $12-15 \mathrm{~Hz}$ or $14-17 \mathrm{~Hz}$. The FZ-A1 protocol was added because of the aforementioned role of the dorsal anterior cingulate in regulating the emotional aspect of pain and because of the role of the frontal cortex in executive skills, such as organization, which was one of the patient's complaints. Half of the session was spent on the first protocol and half on the second. The first protocol continued the T3-T4 placement with consistent inhibits and rewards set where the patient reported less anxiety and a greater sense of wellbeing. The FZ placement was chosen to attempt to decrease the fast beta activity. The $14-17 \mathrm{~Hz}$ reward was introduced in an attempt to improve the patient's executive functioning, including her organizational skills. Simultaneous HRV training was integrated into almost every neurofeedback session, except when the patient felt too tired or overwhelmed. The patient was also instructed in hand warming and was loaned a hand thermometer with which to practice at home. Her headaches decreased in frequency, intensity, and duration, even as her anxiety fluctuated. She often felt a headache coming on in the morning, but these were short-lived and did not persist for the entire day, as they had prior to treatment. Her depression problem score on the NPC also decreased; she was not experiencing depression daily, and when she began to feel depressed, the feeling quickly subsided. At the end of these sessions, migraine was rated as 2 , physical anxiety was rated as 2, depression was rated as 1, sleep problems were rated as 2, overeating was rated as 0 , and organization problems were rated as 2 .

Sessions 8-11 involved two-channel training at T3T4 with inhibits at 2-7 Hz and 22-32 $\mathrm{Hz}$ and reward at $7-10 \mathrm{~Hz}$, and F3-F4 with inhibits at $1-7 \mathrm{~Hz}$ and 22-32 Hz with no reward. At the end of these sessions, migraine was rated as 1 , physical anxiety was rated as 2 , depression was rated as 0 , sleep problems were rated as 2 , overeating was rated as 4 , and organization problems were rated as 2 .
Sessions 12-13 combined the following two protocols: T3-T4 with inhibits as $2-7 \mathrm{~Hz}$ and $22-32$ $\mathrm{Hz}$ and a $7-10 \mathrm{~Hz}$ reward, and $\mathrm{F} 3-\mathrm{F} 4$ with inhibits at 2-7 $\mathrm{Hz}$ and $22-32 \mathrm{~Hz}$ and a $10-13 \mathrm{~Hz}$ reward, varying the frontal placements in an attempt to better impact her organizational skills and also to address her depressed mood, given the role of the frontal lobes in depression (Baehr, Rosenfeld, Baehr, \& Earnest, 1999). Half of each session was spent on each protocol. However, the patient did not experience any additional relief, so passive infrared hemoencephalography ( $\mathrm{pIR} H E G$ ) training was introduced.

Sessions 14-21 alternated between pIR HEG training and EEG neurofeedback within each session. During the neurofeedback training, some additional protocols were introduced due to the increased variability of the patient's headaches and other symptoms. Half of each EEG neurofeedback session was spent with either T3-T4 or C3-C4 with inhibits at 2-7 Hz and $22-32 \mathrm{~Hz}$ and reward at $7-10$ $\mathrm{Hz}$. Half of the session was spent at Fp1-A1, with a 4-8 Hz inhibit and a 15-18 Hz reward. The reward band at Fp1 was introduced in an attempt to activate the inhibitory capacity of the prefrontal cortex. At the beginning of this series of sessions, the patient's headache would start in the morning, but would stop before noon and not return for the rest of the day. Then, in 1 week, she experienced migraine headaches for 4 days, lasting all day, with the pain level at 5 on a 1-10 numerical scale. She rated her migraine problem between 0 and 3.5 on the NPC before the treatment sessions during this period. Her ratings of the overeating problem were also highly variable, ranging from 0 to 3 . Her depression was rated as 0 , physical anxiety was rated between 1 and 3, problems with sleep were rated between 1 and 3 , and organization was rated between 2 and 3 .

For sessions 22-25, the patient was trained at T3-T4 with inhibits of $2-7 \mathrm{~Hz}$ and $22-32 \mathrm{~Hz}$ and reward at $7-10 \mathrm{~Hz}$. During this period, her migraine pain varied from 0 to 3 . At the end of this period, physical anxiety was rated as 1 , depression was rated as 0 , sleep problems were rated as 1 , overeating was rated as 0 , and organization problems were rated as 2.

Sessions 26-32 were conducted at F3-F4 with inhibits at $2-7 \mathrm{~Hz}$ and $22-32 \mathrm{~Hz}$ and a $10-13 \mathrm{~Hz}$ reward to concentrate more on training the frontal cortex. The patient also reported that she had started taking a beta blocker. The patient's rating was 0 for migraines, 1 for physical anxiety, 0 for depression, 0 for sleep, 0.5 for overeating, and 2 for 
organization. On the HRV monitor, her heart rate was lower and more consistent.

\section{Summary of treatment and behaviors outside of the treatment sessions}

This patient presented with migraine symptoms and complications in her mood and family situation. Although EEG neurofeedback approaches provided at the start of treatment appeared to be associated with some initial improvements in pain and other symptoms, there was variability in her symptom intensity over the course of treatment. She also did not experience as much relief with the neurofeedback training as she had hoped, and felt that her functioning continued to be impaired. Rather than persist in continuing to treat her solely with EEG neurofeedback, the decision was made to introduce and include the complementary modalities of HRV (used consistently beginning with session 4) and pIR HEG training (used intermittently beginning with session 14), both of which seemed to provide enhanced relief for the patient. An additional confound was the patient's decision to start a beta blocker prior to the final two sessions. In addition, the patient and the therapist discussed the patient's family stresses and strategies for dealing with them throughout treatment. Overall, there was some improvement in the problems she identified prior to treatment. Specifically, the patient's symptom ratings decreased as follows: migraines, from 4 to 0 ; physical anxiety, from 4 to 1 ; depression, from 4 to 0 ; sleep problems, from 4 to 0 ; overeating, from 4 to 0.5 ; and problems organizing, from 3 to 2 . However, despite these improvements in the problem ratings, the patient reported that she was not satisfied with the outcome, perhaps due to an attribution of the bulk of these improvements to the beta blocker she initiated just before the final two sessions.

\section{Case 4}

\section{Presenting problem}

A 47-year-old divorced man, living with his female partner, presented with daily, severe gastrointestinal pain and diarrhea several times a day, starting between 3 and 5 a.m. and persisting throughout the day. These symptoms caused the patient extreme discomfort and disrupted his work routines and responsibilities. The man worked two jobs as a mechanic, which left him only 1 hour to sleep each day. He further reported, "I have no energy and I'm forgetful." He said, "I can't keep food in me," and said that he had "constant diarrhea." He reportedly ate a healthy diet. The patient had been in talk therapy previously and had not found it to be helpful; he was not interested in engaging in that form of treatment. Prior to treatment, the patient identified the following two areas of concern: diarrhea and pain (rated as a 4) and lack of ability to focus (rated as a 3 ).

\section{History}

The patient reported that the pain and diarrhea started to afflict him daily 14 years earlier. Prior to that, he was experiencing these symptoms more episodically. His symptoms started during a period of time when he was living with his mother and his mother moved frequently because she "got bored." He experienced some remittance of his symptoms one year prior to intake when he went on a plantbased diet for 2 months. His diarrhea decreased to two incidents a month. He went off the diet and was fine for 4 months, but then his symptoms returned to their previous levels.

His surgical history included an appendectomy at age 5 , two surgeries on his right shoulder, surgery on his right knee, and surgery to repair a hernia. He reported a history of pain in his lower back, hips, and knees. He consumed two to three cola beverages on weekends, but denied consuming alcohol, tobacco, or recreational drugs. Family history was significant for Chron's Disease in the patient's older brother and Bipolar Disorder in his younger brother. The patient denied any history of childhood trauma or illness.

\section{Course of treatment}

The patient was seen in neurofeedback treatment, first twice weekly and then once weekly, for 26 sessions. For sessions 1-14, a bipolar placement at T3-T4 was used, because the patient's arousal pattern was not clear and his symptoms seemed indicative of an unstable pattern. The inhibits were at $4-7 \mathrm{~Hz}$ and $18-32 \mathrm{~Hz}$, and the reward bands were $8-12 \mathrm{~Hz}$ and $15-18 \mathrm{~Hz}$. Within five sessions, his diarrhea and pain rating improved to 0 , and his focus rating improved to a 2 . During the course of the five sessions, the patient also reported a gradual reduction in the number of days in which he was afflicted with pain and diarrhea and a decrease in the frequency, intensity, and duration of his symptoms during the days when he was symptomatic. He reported that his mood was better and that he was getting more and better sleep, and had been able to sleep for 5 hours at night. At the eighth session, the patient reported that he had had 6 consecutive symptom-free days, followed by a single day of discomfort, 2 symptom-free days, and then another single day of discomfort. At the eleventh session, the patient reported only one incident of symptom occurrence, without an 
identified precipitating event, with the symptoms lasting 3 hours in the morning.

The decision was jointly made to decrease the frequency of sessions to once per week. At the twelfth session, the patient reported only 1 day of symptoms in the previous week. There followed an interruption of treatment for 3 weeks, due to scheduling difficulties associated with the winter holiday season. When the patient returned, he reported that he had been symptom free until New Year's Eve, when he overate and had 3 days of diarrhea. Since then, however, he had not had gastrointestinal problems. He also reported that his sleep and his memory had worsened in the intervening period. His diarrhea and pain problem was rated as 0 , and his cognitive focus problem was rated as 2 .

For sessions 15-16, we agreed to change the protocol to more directly address the focus problem. We began the protocol CZ-A1 with inhibits at $4-7 \mathrm{~Hz}$ and $22-32 \mathrm{~Hz}$, and the reward band at $15-18 \mathrm{~Hz}$. For session 16, the reward band was increased to 16-19 Hz. After session 16, the patient reported that his diarrhea and pain had worsened and that he was now experiencing these symptoms every morning and after each meal. However, these symptoms were not persisting constantly throughout each day, as they had prior to treatment, and his pain continued to be less intense than previously. He still rated his diarrhea and pain as 0 and focus as 2 . We scheduled a second session that week and, at session 17, we decided to train at T3-T4 with inhibits at $4-7 \mathrm{~Hz}$ and $18-21 \mathrm{~Hz}$ and rewards at $8-12 \mathrm{~Hz}$ and 12-15 Hz. At session 18, the patient reported that he had been symptom free since session 17 , and the decision was made to train half the session at T3-T4 and half the session at CZ-A1. For sessions 18-21, we continued with the T3-T4 protocol for half the session and added CZ-A1, inhibiting $4-7 \mathrm{~Hz}$ and 22-32 Hz and rewarding $16-19 \mathrm{~Hz}$ to improve focus. At session 19, the patient reported experiencing diarrhea again that morning. At session 20, the patient reported having had no diarrhea and pain, but was increasingly troubled by forgetfulness. He understood that this latter problem might be related to his lack of sleep. His diarrhea and pain symptoms remained a 0 on the NPC, but his forgetfulness (not rated on the NPC, because this was not identified as a problem initially) did not improve, so the decision was therefore made to discontinue training at CZ-A1 and to initiate training at FZ-A1 with the same inhibits and rewards, in order to see if that protocol could be more beneficial to his cognitive functioning. This was done for sessions 22-26. By session 24 the patient was reporting improved sleep with more energy, with no resumption of his diarrhea and pain. He reported experiencing bursts of increased energy. This combined protocol had been continued through session 24. At that point, the patient's diarrhea and pain was rated 0 , and his focus was rated 1.5. At session 25, the reward on the FZ-A1 protocol was increased to $17-20 \mathrm{~Hz}$. His diarrhea and pain were rated as 0 , and focus was 1.5 . At session 26 , the patient reported that he had experienced explosive diarrhea all day at work from Saturday through Tuesday. He rated his diarrhea and pain as 4, and focus as 1.5. The training was changed back to the T3-T4 protocol for the entire session.

The patient called the next week to cancel his appointment because of an illness that, he said, had been coming on for the past week or two. The patient then missed his next scheduled appointment. Attempts were made to reach the patient by telephone but the calls were not returned. Two months later, the patient's partner called to report that their relationship had deteriorated and that the patient was very angry, depressed, passiveaggressive and non-communicative. She said that his diarrhea and pain had returned on a daily basis. At a follow-up appointment with the patient and his partner, the patient reported that he did not want to continue with neurofeedback training.

\section{Summary of treatment and behaviors outside of the treatment sessions}

This patient presented with diarrhea and severe gastrointestinal pain of over 14 years duration, with symptoms occurring daily and throughout each day for the past 14 years with a reduction in frequency for several months after he had changed his diet. $\mathrm{He}$ also complained of problems focusing his attention. During treatment, he identified a third problem area (memory problems) that he attributed to lack of sleep and to working two full-time jobs. The patient experienced a gradual but rapid resolution of his diarrhea and pain symptoms over the first five sessions covering 2 weeks of training. Attempts to reduce the frequency of his training were met with a partial resumption of symptoms, but these resolved over time. $\mathrm{He}$ also reported improvements in his ability to focus and improved sleep and mood after the introduction of a protocol that targeted those areas of functioning. By the end of the 25th session, the patient's diarrhea and pain had resolved for several weeks and his cognitive functioning had improved. His diarrhea and pain rating had improved from 4 to 0 , and his problems focusing rating had improved from 3 to 1.5 . 
However at the 26th session, the patient reported a resumption of his symptoms during most days of the preceding week. He later reported that he had been coming down with an illness. The patient cancelled the next session due to illness and missed the appointment after that. He then did not return phone calls. A phone call from the patient's significant other indicated that his symptoms had returned to their previous level and that there was significant conflict in their relationship. At a follow-up session, the patient said that he was not interested in additional neurofeedback training.

\section{Discussion}

These case studies document the improvement in chronic pain symptoms and related improvement in quality of life for patients of various ages, with a variety of chronic pain conditions, who underwent neurofeedback training. All patients had previously undergone conventional medical treatments for their conditions, and two had been in some form of psychological therapy. All of the patients reported substantial symptom relief after the neurofeedback training. However, one of these patients expressed dissatisfaction with the training and attributed her improvement (in headaches) to a beta blocker medication that was initiated towards the end of treatment, even though she had reported substantial improvements in her symptoms prior to this. A second patient who had made significant improvement had a relapse during treatmentdeterioration in his condition, which might have been related to an incipient illness-and his symptoms continued following this relapse. He also elected to discontinue treatment, even though he had reported complete relief from his pain and gastrointestinal symptoms to an extent that he had not experienced in years. Whether he would again experience symptom improvements had he continued with treatment, and whether those improvements would have maintained after any additional treatment was completed, cannot be known.

Although the cortical training sites, and changes in those sites over the course of treatment, were tailored for each patient based on the treatment goals and the patients' response (see summary of protocols in Table 1), treatment protocols common to all four patients included inhibiting theta $(4-7 \mathrm{~Hz})$ and fast beta $(22-32 \mathrm{~Hz})$ activity and rewarding alpha $(8-12 \mathrm{~Hz})$ and slow beta $(12-15 \mathrm{~Hz})$ activity. All four patients were trained at more than one cortical site during the course of the training. These sites included prefrontal, frontal, central, temporal, parietal, and occipital placements; although all four of the patients trained at frontal sites at some point. Three of the patients also trained at temporal sites.

These promising preliminary findings indicate that additional research to study the efficacy of neurofeedback for chronic pain is warranted. Based on these findings, and to the extent that research in this area requires standardization, researchers would do well to consider protocols that include the common elements of the training provided to the patients in this case series-that is, protocols that include inhibition of theta and fast beta and rewarding of alpha and slow beta, with variable training sites. Research comparing these protocols to standard care would be important. It would also be important to assess the effects of nonspecific aspects of the training. This could potentially be done in an experimental study in which one group of subjects receives training with the rewards and inhibits stated above, and another group receives training in a control condition not expected to impact pain.

The selection of the control protocol in a clinical trial would need to take some thought, however. From a scientific perspective, perhaps a "negative protocol" in which theta and fast beta are rewarded and alpha and slow beta are inhibited would be ideal, as this represents the opposite of a protocol hypothesized to be beneficial. However, because of concerns that the negative protocol might have negative effects-a possibility that requires use of such a protocol to confirm-in our view if such a protocol were used, it should be provided for only brief periods of time. Also, in designs using such a protocol, it would be important, from an ethical perspective, for the treatment protocol to be made available to the subjects following the control protocol and for appropriate informed consent and human subjects review to be implemented to protect the subjects from the possible negative effects of the control protocol. 
Table 1

Summary of Neurofeedback Protocols and Responses for Each Case

\begin{tabular}{|c|c|c|c|c|c|}
\hline Patient & Session \# & Training Site(s) & Reward(s) (Hz) & Inhibit(s) (Hz) & Pain intensity \\
\hline \multirow{9}{*}{$\begin{array}{l}\text { \#1 19-year-old female } \\
\text { with GI pain }\end{array}$} & Pre-tx & & & & 4 \\
\hline & $1-3$ & T3-T4 & $\begin{array}{l}\text { Frequency ranging from } \\
\qquad 9-12 \text { to } 12-15\end{array}$ & $2-7,22-32$ & 4 \\
\hline & $6-10$ & FZ-A1 & $8-13$ & $4-7,22-32$ & 2 \\
\hline & $11-18$ & FZ-A1 & $8-13,12-15$ & $4-7,22-32$ & 1 \\
\hline & 20 & P4-A2 & $8-13$ & $4-7,22-32$ & 3 \\
\hline & 21 & FZ-A1 & $8-13,12-15$ & $2-7,22-32$ & \\
\hline & $22-29$ & C4-A2 & $8-13,12-15$ & $2-7,22-32$ & 2 \\
\hline & $30-33$ & 2 channel: F1-A1, F2-A2 & $8-13$ & $4-7,15-32$ & 1 \\
\hline & $34-41$ & 2 channel: F1-A1, F2-A2 & $8-13$ & $0-7,15-32$ & 0 \\
\hline \multirow{7}{*}{$\begin{array}{l}\text { \#2 14-year-old male } \\
\text { with testicular pain }\end{array}$} & & FZ-A1 & $12-15$ or $14-17$ & $2-7,22-32$ & \\
\hline & $8-11$ & $\begin{array}{l}2 \text { channel: T3-T4 } \\
\text { FZ-A1 }\end{array}$ & $\begin{array}{l}7-10 \\
\text { No reward }\end{array}$ & $\begin{array}{l}2-7,22-32 \\
1-7,22-32\end{array}$ & 1 \\
\hline & & T3-T4 & $7-10$ & $2-7,22-32$ & \\
\hline & $12-13$ & F3-F4 & $10-13$ & $2-7,22-32$ & \\
\hline & $14-21$ & $\begin{array}{c}\text { PIR HEG or } \\
\text { T3-T4 or C3-C4 }\end{array}$ & $7-10$ & $2-7,22-32$ & $0-3.5$ \\
\hline & $22-25$ & T3-T4 & $7-10$ & $2-7,22-32$ & $0-3$ \\
\hline & $26-32$ & F3-F4 & $10-13$ & $2-7,22-32$ & 0 \\
\hline \multirow{5}{*}{$\begin{array}{l}\text { \#3 56-year-old female } \\
\text { with migraines }\end{array}$} & Pre-tx & & & & 4 \\
\hline & $12-13$ & F3-F4 & $10-13$ & $2-7,22-32$ & \\
\hline & $14-21$ & $\begin{array}{c}\text { pIR HEG or } \\
\text { T3-T4 or C3-C4 }\end{array}$ & $7-10$ & $2-7,22-32$ & $0-3.5$ \\
\hline & $22-25$ & T3-T4 & $7-10$ & $2-7,22-32$ & $0-3$ \\
\hline & $26-32$ & F3-F4 & $10-13$ & $2-7,22-32$ & 0 \\
\hline \#4 47-year-old male & Pre-tx & & & & 4 \\
\hline \multirow[t]{9}{*}{ with GI pain } & $1-14$ & T3-T4 & $8-12,12-15$ & $4-7,18-32$ & 0 \\
\hline & $15-16$ & CZ-A1 & $15-18$ & $4-7,22-32$ & 0 \\
\hline & 17 & T3-T4 & $8-12,12-15$ & $4-7,22-32$ & \\
\hline & $18-21$ & CZ-A1 & $16-19$ & $4-7,22-32$ & 0 \\
\hline & $10-21$ & T3-T4 & $8-12,12-15$ & $4-7,22-32$ & 0 \\
\hline & $22-24$ & $\begin{array}{l}\text { FZ-A1 } \\
\text { T3-T4 }\end{array}$ & $\begin{array}{c}16-19 \\
8-12,12-15\end{array}$ & $\begin{array}{l}4-7,22-32 \\
4-7,22-32\end{array}$ & 0 \\
\hline & 25 & FZ-A1 & $17-20$ & $4-7,22-32$ & 0 \\
\hline & 20 & T3-T4 & $8-12,12-15$ & $4-7,22-32$ & 0 \\
\hline & 26 & T3-T4 & $8-12,12-15$ & $4-7,22-32$ & 4 \\
\hline
\end{tabular}


A second type of control protocol would be one that might be hypothesized to result in some general benefits (e.g., increased ability to concentrate, improved mood), but that would not necessarily be expected to have direct beneficial effects on pain (for example, a protocol involving rewarding 15-18 $\mathrm{Hz}$ activity, and inhibiting 4-7 Hz and 22-32 Hz activity as measured at $\mathrm{CZ}$ ). While detecting an effect of an "active" (focused on pain reduction) protocol over such a "pain neutral" protocol might be more difficult than detecting an effect over a negative protocol because of indirect beneficial effects of general improvements in mood on pain, such a design might also be considered more ethical; especially, if a negative protocol is indeed found to have negative effects on pain or function. Pilot research to explore the effects of various protocols on outcomes would be useful prior to finalizing any specific design.

\section{Limitations}

This study has a number of important limitations that need to be considered when interpreting the results. Primary among them, of course, is that the study is an uncontrolled case series. Thus, it is not possible to draw conclusions regarding the factors that contributed to the benefits observed. For any one patient, the benefits observed could have been due to time effects or any one or more of many nonspecific factors associated with treatment (e.g., patient or clinician motivations and expectations, therapeutic rapport) or even specific factors (e.g., initiation of a beta blocker) not associated with the neurofeedback treatment. Properly controlled clinical trials are needed to determine which of the benefits that occur with neurofeedback are specific to the neurofeedback training, including the specific training protocol used.

A second important issue is that protocol selection was made based on research on the very limited frequency bands and cortical sites that have been shown to be responsive to pain perception, as well as on research and publications on neurofeedback with other populations. It is possible that other protocols might have been even more effective than the protocols used here. Given the wide variety of chronic pain conditions, including the multiplicity of symptoms, precipitating conditions, levels of intensity, and types of coexisting emotional and behavioral impairment, there is a need for a more systematic process of protocol selection, which only more extensive, controlled research can provide.

A third issue is that the improvements in the patients' conditions were recorded using a general rating of how much of a problem each of the symptoms was in each patient's life. Although this allowed the patients a great deal of latitude to judge for him or herself the impact of their pain, more targeted and specific measures of pain and pain interference might have allowed for a better ability to compare progress and treatment effect across patients. Future researchers in this area-including clinicians who plan to present the results of their clinical work as case studies-would do well to consider using standardized measures of pain and pain-related outcomes in their work (Dworkin et al., 2005; Jensen, 2010; Jensen, Karoly, \& Braver, 1986).

A fourth concern is that decisions to change protocols were based on the clinician's interpretations of the patients' responses, rather than on objective or prearranged criteria. While a large part of the decision making in neurofeedback must be clinical in nature, the lack of any systematically applied criteria for changing protocols results in uncertainty as to whether any one protocol was used for a long enough period of time to see if it was effective. A related point is whether a protocol change was the primary factor in symptom reduction of if the treatment effect was a cumulative effect of all of the protocols used. In case 1, for example, the patient began to demonstrate improvement after an alpha reward was introduced in a certain protocol starting in session 6 . It would be important to further investigate whether this was an essential factor in the patient's improvement.

An additional limitation of the current study was the lack of assessment of EEG activity, as an outcome or process variable, given that the model underlying the use of neurofeedback for pain management hypothesizes a causal role for brain activity in general, and brain oscillations in particular, in the experience of pain (Jensen, Day, \& Miró, 2014). Future researchers (and clinicians presenting case studies) would do well to use the results of qEEGs to help understand the extent to which improvements in pain are accompanied by changes in brain oscillation activity when possible. It is possible that such research could also be used to guide protocol selection and could lead to more effective and efficient treatment.

\section{Summary and Conclusions}

Four patients with a variety of chronic pain conditions were treated with multiple (between 22 and 41) sessions of neurofeedback training. All reported substantial reductions in chronic pain intensity and improvements in other domains of 
quality of life during treatment. However, one patient reported dissatisfaction with the treatment and attributed her improvements to a medication that was initiated towards the end of neurofeedback training, and a second patient relapsed following a brief illness and an interruption in treatment, and subsequently discontinued treatment. The findings from this case series provide additional evidence suggesting that neurofeedback might be an effective method for helping some (and perhaps many) individuals with chronic pain learn self-management skills that would give them more control over, and alleviate, their suffering, and also result in improvements in other related symptoms and problems. This latter point is supported by the findings of Choobforoushzadeh, Neshat-Doost, Molavi, and Abedi (2015), who found that neurofeedback reduced symptoms of depression and fatigue in patients with multiple sclerosis. More research is also warranted to evaluate the efficacy of neurofeedback, relative to other pain treatments, to distinguish the specific from the nonspecific effects of neurofeedback, and to identify the training protocols that are most effective for patients with different pain conditions.

\section{References}

Albert, A. O., Andrasik, F., Moore, J. L., \& Dunn, B. R. (1998). Theta/beta training for attention, concentration and memory improvement in the geriatric population. Applied Psychophysiology and Biofeedback, 23(2), 109.

Andreychuk, T., \& Skriver, C. (1975). Hypnosis and biofeedback in the treatment of migraine headache. International Journal of Clinical and Experimental Hypnosis, 23(3), 172-183.

Apkarian, A. V., Bushnell, M. C., Treede, R.-D., \& Zubeita, J.-K. (2005). Human brain mechanisms of pain perception and regulation in health and disease. European Journal of Pain, 9(4), 463-484. http://dx.doi.org/10.1016/j.ejpain.2004.11.001

Arns, M., Conners, C. K., \& Kraemer, H. C. (2013). A decade of EEG theta/beta ratio research in ADHD: A meta-analysis. Journal of Attention Disorders, 17(5), 374-383. http://dx.doi.org/10.1177/1087054712460087

Arns, M., de Ridder, S., Strehl, U., Breteler, M., \& Coenen, A. (2009). Efficacy of neurofeedback treatment in ADHD: The effects on inattention, impulsivity and hyperactivity: A metaanalysis. Clinical EEG and Neuroscience, 40(3), 180-189. http://dx.doi.org/10.1177/155005940904000311

Arns, M., Heinrich, H., \& Strehl, U. (2014). Evaluation of neurofeedback in ADHD: The long and winding road. Biological Psychology, 95, 108-115. http://dx.doi.org/10.1016/j.biopsycho.2013.11.013

Babiloni, C., Brancucci, A., Babiloni, F., Capotosto, P., Carducci, F., Cincotti, F., ... Rossini, P. M. (2003). Anticipatory cortical responses during the expectancy of a predictable painful stimulation. A high-resolution electroencephalography study. European Journal of Neuroscience, 18(6), 1692-1700. http://dx.doi.org/10.1046/j.1460-9568.2003.02851.x

Babiloni, C., Capotosto, P., Brancucci, A., Del Percio, C., Petrini, L., Buttiglione, M., ... Arendt-Nielsen, L. (2008). Cortical alpha rhythms are related to the anticipation of sensorimotor interaction between painful stimuli and movements: A high- resolution EEG study. The Journal of Pain, 9(10), 902-911. http://dx.doi.org/10.1016/j.jpain.2008.05.007

Baehr, E., Rosenfeld, J. P., Baehr, R., \& Earnest, C. (1999). Clinical use of an alpha asymmetry neurofeedback protocol in the treatment of mood disorders. In J. R. Evans, \& A. Arbarbanel (Eds.), Introduction to Quantitative EEG and Neurofeedback (pp. 181-203). New York: Academic Press.

Bromm, B., \& Lorenz, J. (1998). Neurophysiological evaluation of pain. Electroencephalography and Clinical Neurophysiology, 107(4), 227-253. http://dx.doi.org/10.1016/S0013-4694(98)00075-3

Burroughs, R. D. (2011). Quantitative EEG Analysis of Patients with Chronic Pain: An Exploratory Study. (Master of Science thesis). Retrieved from University of North Texas (UNT) Digital Library, Denton, Texas. http://digital.library.unt.edu/ark:/67531/metadc103294

Chen, A. C. N. (2001). New perspectives in EEG/MEG brain mapping and PET/fMRI neuroimaging of human pain. International Journal of Psychophysiology, 42(2), 147-159. http://dx.doi.org/10.1016/S0167-8760(01)00163-5

Choobforoushzadeh, A., Neshat-Doost, H. T., Molavi, H., \& Abedi, M. R. (2015). Effect of neurofeedback training on depression and fatigue in patients with multiple sclerosis. Applied Psychophysiology and Biofeedback, 40(1), 1-8. http://dx.doi.org/10.1007/s10484-014-9267-4

Cohen, M. J., McArthur, D. L., \& Rickles, W. H. (1980). Comparison of four biofeedback treatments for migraine headache: physiological and headache variables. Psychosomatic Medicine, 42(5), 463-480.

Craig, A. D. (2003a). Interoception: The sense of physiological condition of the body. Current Opinion in Neurobiology, 13(4), 500-505. http://dx.doi.org/10.1016/S0959-4388(03)00090-4

Craig, A. D. (2003b). Pain mechanisms: Labeled lines versus convergence in central processing. Annual Review of Neuroscience, 26, 1-30. http://dx.doi.org/ 10.1146/annurev.neuro.26.041002.131022

Cunningham, M., \& Murphy, P. (1981). The effects of bilateral EEG biofeedback on verbal, visuospatial and creative skills in LD male adolescents. Journal of Learning Disabilities, 14(4), 204-208.

DeCharmes, R. C., Maeda, F., Glover, G. H., Ludlow, D., Pauly, J. M., Soneji, D., ... Mackey, S. C. (2005). Control over brain activation and pain learned by using real-time functional fMRI. Proceedings of the National Academy of Sciences of the United States of America, 102(51), 18626-18631. http://dx.doi.org/10.1073/pnas.0505210102

DeLeo, J. A. (2006). Basic science of pain. The Journal of Bone and Joint Surgery, 88(Suppl. 2), 58-62. http://dx.doi.org/10.2106/JBJS.E.01286

Dworkin, R. H., Turk, D. C., Farrar, J. T., Haythornthwaite, J. A., Jensen, M. P., Katz, N. P., ... Winter, J. (2005). Core outcome measures for chronic pain clinical trials: IMMPACT recommendations. Pain, 113(1-2), 9-19. http://dx.doi.org/10.1016/j.pain.2004.09.012

Egner, T., \& Gruzelier, J. H. (2003). Ecological validity of neurofeedback: Modulation of slow wave EEG enhances musical performance. NeuroReport, 14(9), 1221-1224. http://dx.doi.org/10.1097/00001756-200307010-00006

Escolano, C., Navarro-Gil, M., Garcia-Campayo, J., Congedo, M., \& Minguez, J. (2014). The effects of individual upper alpha neurofeedback in ADHD: An open-label pilot study. Applied Psychophysiology and Biofeedback, 39(3-4), 193-202. http://dx.doi.org/10.1007/s10484-014-9257-6

Fenger, T. N. (1995, September). Visual-motor integration and its relation to EEG neurofeedback brain wave patterns, reading, spelling, and arithmetic achievement in attention deficit disorders and learning disabled students. Paper presented at the meeting of the Society for the Study of Neuronal Regulation, Scottsdale, AZ. 
Flor, H. (2003). Cortical reorganisation and chronic pain: Implications for rehabilitation. Journal of Rehabilitation Medicine, 35(41), 66-72. http://dx.doi.org/10.1080/16501960310010179

Gannon, L., \& Sternbach, R. A. (1971). Alpha enhancement as a treatment for pain: A case study. Journal of Behavior Therapy and Experimental Psychiatry, 2, 209-213.

Gruzelier, J., Egner, T., \& Vernon, D. (2006). Validating the efficacy of neurofeedback for optimizing performance. Progress in Brain Research, 159, 421-431.

Gruzelier, J. (2009). A theory of alpha/theta neurofeedback, creative performance enhancement, long distance functional connectivity and psychological integration. Cognitive Processing, 10(Suppl. 1), 101-109. http://dx.doi.org/10.1007/s10339-008-0248-5

Jensen, M. P. (2010). A neuropsychological model of pain: Research and clinical implications. The Journal of Pain, 11(1), 2-12. http://dx.doi.org/10.1016/j.jpain.2009.05.001

Jensen, M. P., Grierson, C., Tracy-Smith, V., Bacigalupi, S. C., \& Othmer, S. (2007). Neurofeedback treatment for pain associated with complex regional pain syndrome type I. Journal of Neurotherapy, 11(1), 45-53. http://dx.doi.org/10.1300/J184v11n01_04

Jensen, M. P., Karoly, P., \& Braver, S. (1986). The measurement of clinical pain intensity: A comparison of six methods. Pain, 27(1), 117-126. http://dx.doi.org/10.1016/03043959(86)90228-9

Jensen, M. P., Sherlin, L. H., Hakimian, S., \& Fregni, F. (2009). Neuromodulatory approaches for chronic pain management: Research findings and clinical implications. Journal of Neurotherapy, 13(4), 196-213. http://dx.doi.org/10.1080/10874200903334371

Jensen, M. P., Day, M. A., \& Miró, J. (2014). Neuromodulatory treatments for chronic pain: Efficacy and mechanisms. Nature Reviews Neurology, 10, 167-178. http://dx.doi.org/10.1038/nrneurol.2014.12

Katz, J., \& Melzack, R. (1990). Pain "memories" in phantom limbs: Review and clinical observations. Pain, 43, 319-336.

Katz, W. A., \& Rothenberg, R. (2005). The nature of pain: Pathophysiology. Journal of Clinical Rheumatology, 11(2 Suppl.), S11-S15. http://dx.doi.org/10.1097/01.rhu.0000158686.43637.af

Lansbergen, M. M., van Dongen-Boomsma, M., Buitelaar, J. K., \& Slaats-Willemse, D. (2011). ADHD and EEG-neurofeedback: A double-blind randomized placebo-controlled feasibility study. Journal of Neural Transmission, 118(2), 275-284. http://dx.doi.org/10.1007/s00702-010-0524-2

Linden, M., Habib, T., \& Radojevic, V. (1996). A controlled study of the effects of EEG biofeedback on cognition and behavior of children with attention deficit disorder and learning disabilities. Biofeedback and Self-Regulation, 21(1), 35-49.

Lindsay, P., \& Wyckoff, M. (1981). The depression-pain syndrome and its response to antidepressants. Psychosomatics, 22(7), 571-577.

Logemann, H. N. A., Lansbergen, M. M., Van Os, T. W. D. P., Böcker, K. B. E., \& Kenemans, J. L. (2010). The effectiveness of EEG-feedback on attention, impulsivity and EEG: A sham feedback controlled study. Neuroscience Letters, 479(1), 4953. http://dx.doi.org/10.1016/j.neulet.2010.05.026

Lubar, J. F. (1991). Discourse on the development of EEG diagnostics and biofeedback for attention-deficit/hyperactivity disorders. Biofeedback and Self-Regulation, 16(3), 201-225. http://dx.doi.org/10.1007/BF01000016

McCraty, R., Atkinson, M., \& Tomasino, D. (2001). Science of the Heart: Exploring the Role of the Heart in Human Performance (Publication No. 01-001). Boulder Creek, CA: HeartMath Research Center, Institute of HeartMath.
Melzack, R., Coderre, T. J., Katz, J., \& Vaccarino, A. L. (2001). Central neuroplasticity and pathological pain. Annals of the New York Academy of Sciences, 933, 157-174 http://dx.doi.org/10.1111/j.1749-6632.2001.tb05822.x

Melzack, R., \& Perry, C. (1975). Self-regulation of pain: The use of alpha-feedback and hypnotic training for the control of chronic pain. Experimental Neurology, 46, 452-469.

Miltner, W. H. R., \& Weiss, T. (1998). Brain electrical correlates of pain processing. Zeitschrift für Rheumatologie, 57(2 Suppl.), S14-S18. http://dx.doi.org/10.1007/s003930050227

Monastra, V. J., Monastra, D. M., \& George, S. (2002). The effects of stimulant therapy, EEG biofeedback, and parenting style on the primary symptoms of attentiondeficit/hyperactivity disorder. Applied Psychophysiology and Biofeedback, 27(4), 231-249.

Murphy, P. J., Darwin, J., \& Murphy, D. (1977). EEG feedback training for cerebral dysfunction: A research program with learning disabled adolescents. Biofeedback and SelfRegulation, 2, 288.

Nall, A. (1973). Alpha training and the hyperkinetic child-ls it effective? Intervention in School and Clinic, 9(1), 5-19. http://dx.doi.org/10.1177/105345127300900101

National Institute of Neurological Disorders and Stroke, National Institutes of Health. (2015). Pain in America. Retrieved from http://www.ninds.nih.gov/disorders/chronic_pain/chronic_pain. htm

Nishigami, T., Nakano, H., Osumi, M., Tsujishita, M., Mibu, A., \& Ushida, T. (2014). Central neural mechanisms of interindividual difference in discomfort during sensorimotor incongruence in healthy volunteers: An experimental study. Rheumatology, 53(7), 1194-1199. http://dx.doi.org/10.1093/rheumatology/ket494

Othmer, S. F. (2005). Interhemispheric EEG training. Journal of Neurotherapy, 9(2), 87-96. http://dx.doi.org/10.1300/J184v09n02_08

Othmer, S., Othmer, S. F., \& Kaiser, D. A. (1999). EEG biofeedback: Training for $A D / H D$ and related disruptive behavior disorders. Chapter in J. A. Incorvaia, B. S. MarkGoldstein, \& D. Tessmer (Eds.), Understanding, Diagnosing, and Treating $A D / H D$ in Children and Adolescents: $A n$ Integrative Approach (235-296). New York: Jason Aronson Press. Peyron, R., Laurent, B., \& Garcia-Larrea, L. (2000). Functional imaging of brain responses to pain. A review and meta-analysis. Clinical Neurophysiology, 30(5), 263-288.

Pfurtscheller, G. (2003). Induced oscillations in the alpha band: Functional meaning. Epilepsia, 44(Suppl. 12), 2-8. http://dx.doi.org/10.1111/j.0013-9580.2003.12001.x

Prinsloo, S., Gabel, S., Lyle, R., \& Cohen, L. (2012). Neuromodulation of cancer pain. Integrative Cancer Therapies, 13(1), 30-37. http://dx.doi.org/10.1177/1534735413477193

Rainville, P., Duncan, G. H., Price, D. D., Carrier, B., \& Bushnell, M. C. (1997). Pain affect encoded in human anterior cingulate but not in somatosensory cortex. Science, 277(5328), 968971.

Rasey, H. W., Lubar, J. E., Mclntyre, A., Zoffuto, A. C., \& Abbott, P. L. (1996). EEG biofeedback for the enhancement of attentional processing in normal college students. Journal of Neurotherapy, 1(3), 15-21.

Thompson, L., \&, Thompson, M. (1998). Neurofeedback combined with training in metacognitive strategies: Effectiveness in students with ADD. Applied Psychophysiology and Biofeedback, 23(4), 243-263. http://dx.doi.org/10.1023/A:1022213731956

Tinazzi, M., Fiaschi, A., Rosso, T., Faccioli, F., Grosslercher, J., \& Aglioti, S. M. (2000). Neuroplastic changes related to pain occur at multiple levels of the human somatosensory system: A somatosensory-evoked potentials study in patients with cervical radicular pain. The Journal of Neuroscience, 20(24), 9277-9283. 
Vernon, D., Egner, T., Cooper, N., Compton, T., Neilands, C., Sheri, A., \& Gruzelier, J. (2003). The effect of training distinct neurofeedback protocols on aspects of cognitive performance. International Journal of Psychophysiology, 47(1), 75-85.

Whisenant, W. F., \& Murphy, P. J. (1977). Bilateral EEG biofeedback and creativity. Biofeedback and Self-Regulation, 2, 322.

Received: May 11, 2015

Accepted: June 9, 2015

Published: July 15, 2015 